\title{
How to acknowledge hypercomputation?
}

\author{
Alexander Leitsch, Günter Schachner \\ Institut für Computersprachen, Vienna University of Technology, \\ Favoritenstr.9/185, 1040 Vienna, Austria \\ and \\ Karl Svozil ${ }^{\dagger}$ \\ Institute for Theoretical Physics, Vienna University of Technology, \\ Wiedner Hauptstraße 8-10/136, 1040 Vienna, Austria
}

\begin{abstract}
We discuss the question of how to operationally validate whether or not a "hypercomputer" performs better than the known discrete computational models.
\end{abstract}

\section{Introduction}

It is widely acknowledged [1, 2] that every physical system corresponds to a computational process, and that every computational process, if applicable, has to be physically and operationally feasible in some concrete realization. In this sense, the physical and computational capacities should match; because if one is lagging behind the other, there is a lack in the formalism and its potential scientific and ultimately technological applicability. Therefore, the exact correspondence of the mathematical formalism on the one hand, and the particular physical system which is represented by that formalism on the other hand, demands careful attention.

If one insists on operationalizability [3], one needs not go very far in the history of mathematics to encounter suspicious mathematical objects. Surely enough,

\footnotetext{
*leitsch@logic.at

†svozil@tuwien.ac.at
} 
the number $\pi$ can be defined and effectively computed as the ratio of the circumference to the diameter of a "perfect (platonic)" circle. Likewise, the numbers $\sqrt{2}$ and $\sqrt{3}$ can be interpreted as the ratio between the length of the diagonal to the side length of any square and cube. But it is not totally unjustified to ask whether or not these numbers have any operational meaning in a strict physical sense; i.e., whether such numbers could, at least in principle, be constructed and measured with arbitrary or even with absolute precision.

At the heart of most of the problems seems to lie the ancient issue of the "very large/small" or even potential infinite versus the actual infinite. Whereas the mathematical formalism postulates the existence of actual infinite constructions and methods, such as the summation of a (convergent) geometric series, or diagonalization, the physical processes, methods and techniques are never infinite.

Suppose, as an example, one would attempt to operationalize $\pi$. Any construction of a "real" circle and one of its diameters, and a subsequent measurement thereof, would find its natural scale bound from below by the atomistic structure of matter upon which any such circle is based. Long before those molecular or atomic scales, the physical geometry might turn out to be not as straightforward as it appears from larger scales; e.g., the object might turn out to be a fractal.

Chaitin's Omega number [4] is interpretable as the halting probability of a universal computer. Omega is provably uncomputable; i.e., no computer, in any of the (equivalent universal) forms conceptualized so far, "computes" Omega. Thus, only hypercomputers might be capable to "compute" Omega. Yet, Omega can be "computed in the limit" (without any computable radius of convergence) by a finite-size program in infinite time and with infinite space. Just as for many transcendential numbers - the difference being the absence of any computable radius of convergence - the first digits of Omega are well known [5], yet Omega is provable algorithmically incompressible and thus random. Nevertheless, presently, for all practical purposes, the statement that "the $10^{10^{10^{10}}}$ th digit in a decimal expansion of Euler's number $e$ is 5" is as unverifiable as a similar statement for Omega. [Note, however, that for certain transcendental numbers such as Pi, the BaileyBorwein-Plouffe (BBP) formula [6, 7] can be used to efficiently calculate "large" digits in various bases without knowing all of the previous digits.] Omega encodes all decision problems which can be algorithmically interpreted. For instance, for a particular universal computer, Goldbach's conjecture and Riemann's hypothesis could be decided with programs of size 3484 and 7780 bits, respectively [8]. Yet, Omega appears to have two features which are normally considered contradictory: it is one of the most informative mathematical numbers imaginable. Yet at 
the same time this information is so compressed that it cannot be deciphered; thus Omega appears to be totally structureless and random. In this sense, for Omega, total information and total randomness seem to be "two sides of the same coin." On a more pragmatic level, it seems impossible here to differentiate between order and chaos, between knowledge and chance. This gives a taste of what can be expected from any "hypercomputation" beyond universal computability as defined by Turing.

It should always be kept in mind that all our sense perceptions are derived from elementary discrete events, such as clicks in photon or particle detectors, even if they appear to be analog: the apparently smooth behavior has a discrete fine structure. Among other issues, such as finiteness of system resources, this discreteness seems to prohibit the "physical realization" of any actual infinities.

What is the physical meaning of infinite concepts, such as space-time singularities, point particles, or infinite precision? For instance, are infinity machines with geometrically squeezed time cycles, such as the ones envisioned by Weyl [9] and others [10, 11, 12, 13, 14, 15, 16, 17, 18, 19, 20] physically feasible? Motivated by recent proposals to utilize quantum computation for trespassing the Turing barrier [21, 22, 23, 24], these accelerating Turing machines have been intensively discussed [25] among other forms of hypercomputation [26, 27, 28].

Certainly, the almost ruthless and consequential application of seemingly mindboggling theories such as quantum mechanics, as far as finitistic methods are concerned, has yielded one success after another. Nevertheless, the use of actual transfinite concepts and methods remains highly conjectural.

A priori, while it may appear rash to exclude the transfinite in general, and transfinite set theory in particular from physics proper, one should be aware of its counterintuitive consequences, such as for instance the Banach-Tarski paradox, and be careful in claiming its physical applicability. Recall the old phrase attributed to Einstein and Infeld (Ref. [29], p.31), "Physical concepts are free creations of the human mind, and are not, however it may seem, uniquely determined by the external world."

To this point, we are not aware of any test, let alone any application, of the actual transfinite in Nature. While general contemplations about hypercomputations and the applicability of transfinite concepts for physics may appear philosophically interesting, our main concern will be operational testability: if presented with claims that hypercomputers exist, how could we possibly falsify, or even verify and test such propositions [30]?

In what follows, hypercomputation will be conceptualized in terms of a black box with its input/output behavior. Several tests and their rather limited scope 
will be evaluated. Already in 1958, Davis [31, p. 11] sets the stage of the following discussion by pointing out "... how can we ever exclude the possibility of our being presented, some day (perhaps by some extraterrestrial visitors), with a (perhaps extremely complex) device or "oracle" that "computes" a non-computable function?" While this may have been a remote, amusing issue in the days written, the advancement of physical theory in the past decades has made necessary a careful evaluation of the possibilities and options for verification and falsification of certain claims that a concrete physical system "computes" a non-computable function.

\section{On black boxes which are hypercomputers}

Digital computers are "reliable" in the sense of "comprehensible, reproducible, traceable, accountable," just for the reason that the internal functioning of a digital computer is known to anyone seeking such knowledge, and that the programs give a logical specification of an algorithm. Because the code is known one can - at least in principle - verify the behavior for all inputs mathematically.

One cannot expect that hypercomputers will be "reliable" in the aforementioned sense. The alleged "hypercomputer" may be presented as a device, an agent or an oracle of unknown provenance, and in particular with no rational explanation (in the traditional algorithmic sense) of its intrinsic working. (For the sake of an example, consider Laplace being confronted with a modern digital clock.) Therefore, we attempt to deal with hypercomputers purely phenomenologically; i.e., by analyzing their input/output behavior. Thereby, the hypercomputer will be treated as black box with input and output interfaces; see Fig. 1 .

The following notation is introduced. Let $B$ be a subset of $X_{1} \times \ldots \times X_{m}$. The $i$-th projection of $B$ (for $i=1, \ldots, m$ ), written as $B_{i}$, is defined by:

$$
\begin{aligned}
B_{i}= & \left\{x \mid x \in X_{i},\left(\exists y \in X_{1} \times \ldots \times X_{i-1}\right)\left(\exists z \in X_{i+1} \times \cdots \times X_{m}\right)\right. \\
& (y, x, z) \in B\} .
\end{aligned}
$$

For any $x \in N^{m}$ we define

$$
|x|=\max \left\{x_{i} \mid i \in\{1, \ldots, m\}\right\} .
$$

Then, a hypercomputer can be defined via its input/output behavior of black boxes as follows. 


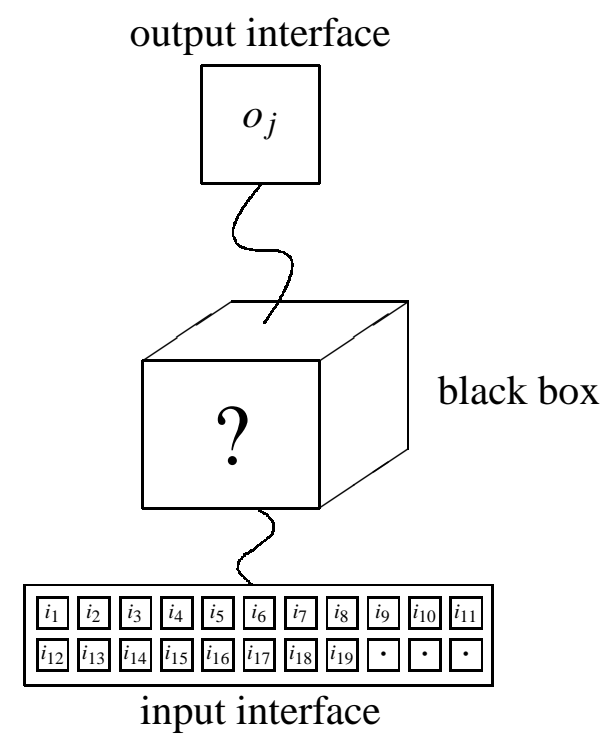

Figure 1: Hypercomputers of unknown origin may be studied phenomenologically; i.e., by considering them as a black box with input and output interfaces, and by studying their input-output behavior.

Definition 1 (black box) Let $X, Y$ be sets and $\mathbb{N}$ be the set of natural numbers. $A$ subset $B$ of $X \times Y \times \mathbb{N}$ is called a black box if $B_{1}=X$. $X$ is called the input set and $Y$ the output set.

Note that the condition $B_{1}=X$ models a computing device which is total, i.e. there exists always an output.

Definition 2 Let $B$ be a black box. We define

$$
\begin{aligned}
f_{B} & =\{(x, y) \mid(\exists z)(x, y, z) \in B\}, \\
t_{B} & =\{(x, z) \mid(\exists y)(x, y, z) \in B\} .
\end{aligned}
$$

$f_{B}$ is called the input-output relation of $B$ and $t_{B}$ the computing time of $B$. If $f_{B}$ and $t_{B}$ are functions then $B$ is called deterministic.

Every halting deterministic Turing machine defines a black box. Indeed, let $M$ be a Turing machine (computing a total function), $f_{M}: X \rightarrow Y$ be the function computed by $M$ and $t_{M}$ be the computing time of $M$. Then

$$
\left\{\left(x, f_{M}(x), t_{M}(x)\right) \mid x \in X\right\}
$$


is a (deterministic) black box. Similarly all halting non-deterministic Turing machines define black boxes.

Definition 3 (hypercomputer) A strong hypercomputer is a black box $B$ where $f_{B}$ is not Turing-computable.

Definition 4 Let $C$ be a class of computable monotone functions $\mathbb{N} \rightarrow \mathbb{N}$ containing the polynomials (over $\mathbb{N}$ with non-negative coefficients). Then $\mathcal{C}$ is called a bound class.

Definition 5 A weak hypercomputer is a black box $B$ with the following property: There exists a bound class $\mathcal{C}$ such that

- $t_{M}(x)>g(|x|)$ almost everywhere for all $g \in \mathcal{C}$ and for all Turing machines $M$ with $f_{M}=f_{B}$.

- There exists an $h \in \mathcal{C}$ such that $t_{B}(x) \leq h(|x|)$ for all $x \in B_{1}$.

A strong hypercomputer computes either a non-computable function or decides an undecidable problem. A weak hypercomputation outperforms all Turing machines. A possible scenario for a weak hypercomputer $B$ is the following: $f_{B}$ is an EXPTIME-complete problem, therefore there exists no polynomial $p$ and no Turing machine $M$ computing $f_{B}$ with $t_{M}(x) \leq p(|x|)$ for all $x \in X$, but $t_{B}(x) \leq p(|x|)$ for all $x \in X$ and for a polynomial $p$.

For non-deterministic hypercomputers we may distinguish between the following cases:

- $f_{B}$ is not a function,

- $f_{B}$ is a function, but $t_{B}$ is not.

For stochastic hypercomputers, either $t_{B}$ or both $f_{B}$ and $t_{B}$ are random variables, and the requirements on the computation have to be specified.

\section{Tests}

Having set the stage for a general investigation into hypercomputers which are presented to us as black boxes, we shall consider a few cases and tests. These test methods will be essentially heuristic and present no way of systematically addressing the issue of falsifying or even verifying hypercomputation. 
One strategy for creating tests will be to consider problems which are asymmetric with respect to their creation and verification - which should be "easy" — on the one hand, and their solution — which should be "hard" — on the other hand.

\subsection{NP-complete cases}

It may be conjectured that, by operational means, it is not possible to go beyond tests of hyper-NP-completeness. Even for an NP-complete problem as for instance the satisfiability problem of propositional logic (SAT), it is not trivial to verify that a hypercomputer solves the problem in polynomial time. Without insight into the internal structure of the hypercomputer we cannot obtain a proof of polynomial time computation, which is an asymptotic result. Even here we rely on experiments to test a "large" number of problems. A central problem consists in the right selection of problem sequences. If the selection is based on random generators we merely obtain results on average complexity, which would not be significant.

Furthermore, we need at least some information about the polynomial in question (e.g., its maximum degree). Otherwise it remains impossible to decide by finite means whether some behavior is polynomial or not.

\subsection{Harder cases with tractable verification}

Do there exist (decision) problems which are harder than the known NP-complete cases, possibly having no recursively enumerable solution and proof methods, whose results nevertheless are tractable verifiable? For example, the problem of graph non-isomorphism (GNI) is one that is not known to be in NP, not even in NP $\cup$ BPP. Nevertheless, it is possible to "efficiently verify" whether a "prover" solves this problem correctly.

If the prover claims that two graphs $G_{1}$ and $G_{2}$ are isomorphic, he can convince us by providing a graph isomorphism. That can be checked in polynomial time, which also means that GNI $\in$ coNP. If, on the other hand, the prover claims that $G_{1}$ and $G_{2}$ are non-isomorphic, we can verify this by the following interactive proof:

1. Choose one of the graphs $G_{1}$ and $G_{2}$ with equal probability.

2. Apply an arbitrary permutation to its vertices; this yields graph $H$. 
3. The prover must decide whether $H$ is equivalent to $G_{1}$ or $G_{2}$.

4. Repeat for $N$ rounds.

If the initial answer was wrong and the graphs $G_{1}$ and $G_{2}$ are actually isomorphic, the prover can in step 3 only guess which graph was chosen in step 1 (since now $H$ could have been derived from either). Hence, after $N$ rounds we can be sure with probability $1-2^{-N}$ that the graphs $G_{1}$ and $G_{2}$ are non-isomorphic.

By denoting the class of interactive proofs by IP, we have shown that GNI $\in$ IP. Interactive proofs further exist for every language in PSPACE (which is assumed to be much larger than NP). In fact, it can be shown [32] that IP equals PSPACE. This means, in particular, that IP is closed under complement.

The protocol in the example above has the property that in each round a constant number of messages is sent. In a generic interactive proof system for PSPACE this is not necessarily true; but at any instance the number of messages depends polynomially on the input length.

In the literature, specific classes of interactive proof systems are investigated as well, e.g. the Arthur-Merlin class [33] and the Goldwasser-Micali-Rackoff(GMR) class [34]. The former uses public coin tosses, with the intention to accommodate certain languages in as low complexity classes as possible. The latter uses private coin tosses, with the intention to cover the widest possible class of efficiently verifiable languages; additionally, it has the feature of providing zero-knowledge proofs, which is of great significance in cryptography. (The protocol presented above does not have the zero-knowledge property - unless GNI $\in$ BPP -, but can be modified to have.) For further information on interactive proof systems see [35, 36].

\subsection{Interference of problems}

One may confront the hypercomputer with the problem of comparing the solutions of multiple tasks. Such a comparison needs not necessarily involve the separate computation of the solutions of these multiple tasks.

As an analogy, consider Deutsch's problem as one of the first problems which quantum computers could solve effectively. Consider a function that takes a single (classical) bit into a single (classical) bit. There are four such functions $f_{1}, \ldots, f_{4}$, corresponding to all variations. One can specify or "prepare" a function bitwise, or alternatively, one may specify it by requiring that, for instance, such a function acquires different values on different inputs, such as $f(0) \neq f(1)$. Thereby, we may, even in principle, learn nothing about the individual functional values alone. 
A related issue is the question of whether or not one hypercomputer should be allowed to verify another hypercomputation. This would be similar to using one algorithm to prove the four color theorem, and, since it is unfeasible for a human to verify by hand, another algorithm to check the validity of the former computer-assisted proof.

\subsection{Generation of random sequences}

By implementation of Chaitin's "algorithm" to compute Chaitin's $\Omega$ [37] or variants thereof [38], it would in principle be possible to "compute" the first bits of random sequences. Such random sequences could in principle be subject to the usual tests of stochasticity [39, 40].

Note that in quantum mechanics, the randomness of certain microphysical events, such as the spontaneous decay of excited quantum states [41, 42], or the quantum coin toss experiments in complete context mismatches [39] is postulated as an axiom. This postulate is then used as the basis for the production of quantum randomness oracles such as the commercially available Quantis $^{\mathrm{TM}}$ interface [43].

\section{Impossibility of unsolvable problems whose "so- lution" is polynomially verifiable}

Let $\Sigma_{0}$ be a finite (non-empty) alphabet and $X \subset \Sigma_{0}^{*}$ be a semi-decidable, but not decidable set. That means there exists a Turing machine which accepts the language $X$, but does not terminate on all $x \in \Sigma_{0}^{*}$. The concept of acceptable by Turing machines is equivalent to derivable by inference systems or producible by grammars. We choose the approach of a universal proof system, i.e. of a system which simulates every Turing machine.

Let $P$ be such a proof system. Let $V$ be an infinite set of variables (over strings in $\Sigma^{*}$ ). A meta-string is an object $x_{1} \ldots x_{n}$ where $x_{i} \in \Sigma$ or $x_{i} \in V$. If $X$ is a metastring and $\theta$ is a substitution (i.e. a mapping $\left.V \rightarrow(V \cup \Sigma)^{*}\right)$ then $X \theta$ is called an instance of $X$. If $X \theta \in \Sigma^{*}$ we call $X \theta$ a ground instance of $X$.

We may define $P=\left(\mathcal{Y}, X, \Sigma, \Sigma_{0}\right)$ where $\mathcal{Y}$ is a finite sets of meta-strings (the axioms) and $X$ is a finite set of rules, i.e. expressions of the form:

$$
\frac{X_{1} \ldots X_{n}}{X}
$$


where $X_{1}, \ldots, X_{n}, X$ are meta-strings such that the set of variables in $X$ is contained in the set of variables in $X_{1}, \ldots, X_{n}$.

$\Sigma_{0}$ is a (non-empty) subset of $\Sigma$ (defining the strings of the theory to be generated).

A derivation $\varphi$ in $P$ is a tree such that all nodes are labelled by strings in $\Sigma^{*}$. In particular:

- the leaves of $\varphi$ are labelled by ground instances of axioms.

- Let $N$ be a node in $\varphi$ which is not a leaf and $\left(N, N_{1}\right), \ldots,\left(N, N_{k}\right)$ be the nodes from $N$ then

$$
\frac{N_{1} \ldots N_{k}}{N}
$$

is a ground instance of a rule in $X$.

A proof of an $x$ in $\Sigma_{0}^{*}$ in $P$ is a derivation in $P$ with the root node labeled by $x$. $x$ is called provable in $P$ if there exists a proof of $x$ in $P$.

fact: as $\Sigma$ is finite there are only finitely many derivations of length $\leq k$ for any natural number $k$, where length is the number of symbol occurrences. Let $P[k]$ be the set of all derivations of length $\leq k$.

We prove now:

There is no recursive function $g$ such that for all $x \in X$ :

(*) $x$ is provable in $P$ iff there exists a proof $\varphi$ of $x$ with $|\varphi| \leq g(|x|)$.

\section{Proof:}

Assume that there exists a recursive $g$ such that $(*)$ holds. We construct a decision procedure of $X$ :

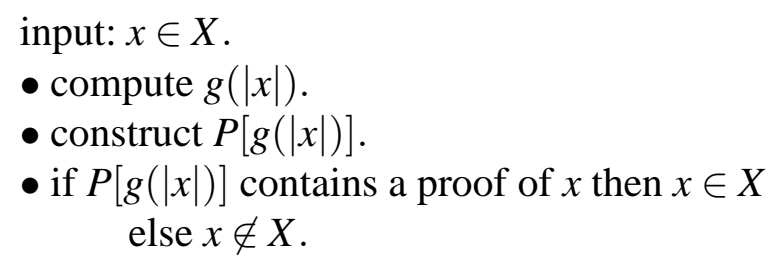

But we assumed $X$ to be undecidable, thus we arrive at a complete contradiction. Q.E.D.

It follows as a corollary that there exists no proof system which generates an undecidable problem $X$ and $X$ is polynomially verifiable. 
The result above illustrates one of the problems in acknowledging hypercomputation. Even if we have a strong hypercomputer solving, let us say, the halting problem, the verification of its correctness is ultimately unfeasible. Due to the absence of recursive bounds we cannot expect to obtain a full proof of the corresponding property (halting or non-halting) from the hypercomputer itself.

When we consider the halting problem and the property of non-halting, this can only be verified by a proof (and not by simulating a Turing machine). By the undecidability of the problem there is no complete (recursive) proof system doing the job. So when we obtain a verification from the hypercomputer concerning non-halting, the form of this verification lies outside computational proof systems.

However we might think about the following test procedure for hypercomputers: humans create a test set of problems for an undecidable problem $X$, i.e. a finite set $Y$ with $Y \cap X \neq \emptyset$ and $Y \cap X^{c} \neq \emptyset$. The humans are in possession of the solutions, preferably of proofs $\varphi_{y}$ of $y \in X$ or of $y \notin X$ for any $y \in Y$. This finite set may at least serve the purpose of falsifying hypercomputation (provided the hypercomputer is not stochastic and wrong answers are admitted). Beyond the possibility of falsification we might consider the following scenario: the hypercomputer answers all questions concerning the test set $Y$ correctly, and its computing time is independent of the complexity of the proofs $\varphi_{y}$. Such a phenomenon would, of course, not yield a verification of the hypercomputer but at least indicate a behavior structurally differing from computable proof systems.

But the ultimate barrier of verifying a hypercomputer is that of verifying a black box, characterized by the attempt to induce a property of infinitely many input-output pairs by a finite test set.

\section{Discussion and summary}

The considerations presented here may be viewed as special cases of a very general black box identification problem: is it possible to deduce certain features of a black box, without screwing the box open and without knowing the intrinsic working of the black box, from its input/output behavior alone? Several issues of this general problem have already been discussed. For instance, in an effort to formalize the uncertainty principle, Moore [44] considered initial state identification problems of (given) deterministic finite automata. Gold [45, 46, 47, 48, 49] considered a question related to induction: if one restricts black boxes to computable functions, then the rule inference problem, i.e., the problem to find out which function is implemented by the black box, is in general unsolvable. The halting 
problem [50, 51, 52] can be translated into a black box problem: given a black box with known partial recursive function, then its future behavior is generally unpredictable. Even the problem to determine whether or not a black box system is polynomial in computation space and time appears to be far from being trivial. In general, finding a way to falsify hypercomputation by a single counterexample may turn out to be easier than proving hypercomputation in full generality.

So, if presented with a hypercomputer or oracle, we could only assert heuristic information, nothing more. We have to accept the fact that more general assertions, or even proofs for computational capacities beyond very limited finite computational capacities remain impossible, and will possibly remain so forever.

The situation is not dissimilar from claims of absolute indeterminism and randomness on a microphysical scale [39], where a few, albeit subtle tests of time

series [40] generated by quantum randomness oracles such as Quantis ${ }^{\mathrm{TM}}$ [43] can be compared against advanced algorithmic random number generators such as the Rule30CA Wolfram rule 30 generator implemented by Mathematica ${ }^{\circledR}$. Beyond heuristic testing, any general statement about quantum randomness remains conjectural.

\section{Acknowledgments}

This manuscript grew out of discussions between computer scientists and physicists at the Vienna University of Technology, including, among others, Erman Acar, Bernhard Gramlich, Markus Moschner, and Gernot Salzer.

\section{References}

[1] S. Wolfram, A New Kind of Science (Wolfram Media, Inc., Champaign, IL, 2002).

[2] K. Svozil, "Computational universes," Chaos, Solitons \& Fractals 25, 845859 (2006). http://dx.doi.org/10.1016/j.chaos.2004.11.055

[3] P. W. Bridgman, "A Physicist's Second Reaction to Mengenlehre," Scripta Mathematica 2, 101-117, 224-234 (1934), cf. R. Landauer [53].

[4] G. J. Chaitin, Algorithmic Information Theory (Cambridge University Press, Cambridge, 1987). 
[5] C. S. Calude and M. J. Dinneen, "Exact Approximations of Omega Numbers," International Journal of Bifurcation and Chaos 17, 1937-1954 (2007), CDMTCS report series 293. http://dx.doi.org/10.1142/S0218127407018130

[6] D. Bailey, P. Borwein, and S. Plouffe, "On the rapid computation of various polylogarithmic constants," Mathematics of Computation 66, 903-913 (1997). http://dx.doi.org/10.1090/S0025-5718-97-00856-9

[7] D. H. Bailey and J. M. Borwein, "Experimental Mathematics: Examples, Methods and Implications," Notices of the American Mathematical Society 52, 502-514 (2005). http://www.ams.org/notices/200505/fea-borwein.pdf

[8] C. S. Calude and E. C. M. J. Dinneen, "A new measure of the difficulty of problems," Journal for Multiple-Valued Logic and Soft Computing 12, 285307 (2006), CDMTCS report series 277. http://www.cs.auckland.ac.nz/CDMTCS//researchreports/277cris.pdf

[9] H. Weyl, Philosophy of Mathematics and Natural Science (Princeton University Press, Princeton, 1949).

[10] A. Grünbaum, Philosophical problems of space and time (Boston Studies in the Philosophy of Science, vol. 12) (D. Reidel, Dordrecht/Boston, 1974), second, enlarged edition edn.

[11] J. F. Thomson, “Tasks and supertasks,” Analysis 15, 1-13 (1954).

[12] P. Benacerraf, "Tasks and supertasks, and the modern Eleatics," Journal of Philosophy LIX, 765-784 (1962).

[13] R. Rucker, Infinity and the Mind (Birkhäuser, Boston, 1982), reprinted by Bantam Books, 1986.

[14] I. Pitowsky, "The physical Church-Turing thesis and physical computational complexity," Iyyun 39, 81-99 (1990).

[15] J. Earman and J. D. Norton, "Forever is a day: supertasks in Pitowsky and Malament-Hogart spacetimes," Philosophy of Science 60, 22-42 (1993). 
[16] M. Hogarth, "Predicting the future in relativistic spacetimes," Studies in History and Philosophy of Science. Studies in History and Philosophy of Modern Physics 24, 721-739 (1993).

[17] M. Hogarth, "Non-Turing computers and non-Turing computability," PSA 1, 126-138 (1994).

[18] E. W. Beth, The Foundations of Metamathematics (North-Holland, Amsterdam, 1959).

[19] E. G. K. López-Escobar, “Zeno’s Paradoxes: Pre Gödelian Incompleteness,” Yearbook 1991 of the Kurt-Gödel-Society 4, 49-63 (1991).

[20] K. Svozil, "The Church-Turing Thesis as a Guiding Principle for Physics," in Unconventional Models of Computation, C. S. Calude, J. Casti, and M. J. Dinneen, eds., pp. 371-385 (1998).

[21] C. S. Calude and B. Pavlov, "Coins, Quantum Measurements, and Turing's Barrier," Quantum Information Processing 1, 107-127 (2002). http://arxiv.org/abs/quant-ph/0112087

[22] V. A. Adamyan and B. S. P. Cristian S. Calude, "Transcending the Limits of Turing Computability," (1998). http://arxiv.org/abs/quant-ph/0304128

[23] T. D. Kieu, "Quantum Algorithm for Hilbert's Tenth Problem," International Journal of Theoretical Physics 42, 1461-1478 (2003). http://arxiv.org/abs/quant-ph/0110136

[24] T. D. Kieu, "Computing the Noncomputable," Contemporary Physics 44, 51-71 (2003). http://arxiv.org/abs/quant-ph/0203034

[25] T. Ord, "The many forms of hypercomputation," Applied Mathematics and Computation 178, 143-153 (2006). http://dx.doi.org/10.1016/j.amc.2005.09.076

[26] M. Davis, "The myth of hypercomputation," in Alan Turing: Life and Legacy of a Great Thinker, C. Teuscher, ed. (Springer, Berlin, 2004), pp. 195-212. 
[27] F. A. Doria and J. F. Costa, "Introduction to the special issue on hypercomputation," Applied Mathematics and Computation 178, 1-3 (2006). http://dx.doi.org/10.1016/j.amc.2005.09.065

[28] M. Davis, "Why there is no such discipline as hypercomputation," Applied Mathematics and Computation 178, 4-7 (2006). http://dx.doi.org/10.1016/j.amc.2005.09.066

[29] A. Einstein and L. Infeld, The evolution of physics (Cambridge University Press, Cambridge, 1938).

[30] T. Y. Chow, "The Myth of Hypercomputation," (2004), contribution to a discussion group on hypercomputation. http://cs.nyu.edu/pipermail/fom/2004-February/007883.html

[31] M. Davis, Computability and Unsolvability (McGraw-Hill, New York, 1958).

[32] A. Shamir, "IP = PSPACE," J. ACM 39, 869-877 (1992). http://dx.doi.org/10.1145/146585.146609

[33] L. Babai, "Trading group theory for randomness," in STOC '85: Proceedings of the seventeenth annual ACM symposium on theory of computing pp. 421429 (1985).

http://dx.doi.org/10.1145/22145.22192

[34] S. Goldwasser, S. Micali, and C. Rackoff, "The knowledge complexity of interactive proof systems," SIAM J. Comput. 18, 186-208 (1989). http://dx.doi.org/10.1137/0218012

[35] L. Babai and S. Moran, "Arthur-Merlin games: A randomized proof system, and a hierarchy of complexity classes," Journal of Comp. and Syst. Sci. 36, 254-276 (1988).

[36] O. Goldreich, Foundations of Cryptography: Basic Tools (Cambridge University Press, Cambridge, 2001).

[37] G. J. Chaitin, Exploring Randomness (Springer Verlag, London, 2001).

[38] C. Calude, Information and Randomness-An Algorithmic Perspective (Springer, Berlin, 1994). 
[39] K. Svozil, "The quantum coin toss-Testing microphysical undecidability," Physics Letters A 143, 433-437 (1990). http://dx.doi.org/10.1016/0375-9601(90)90408-G

[40] C. S. Calude and M. J. Dinneen, "Is quantum randomness algorithmic random? A preliminary attack," in Proceedings 1st International Conference on Algebraic Informatics, S. Bozapalidis, A. Kalampakas, and G. Rahonis, eds., pp. 195-196 (2005).

[41] T. Erber, "Testing the Randomness of Quantum Mechanics: Nature's Ultimate Cryptogram?" in Annals of the New York Academy of Sciences. Volume 755 Fundamental Problems in Quantum Theory, D. M. Greenberger and A. Zeilinger, eds. (Springer, Berlin, Heidelberg, New York, 1995), Vol. 755, pp. 748-756.

http://dx.doi.org/10.1111/j.1749-6632.1995.tb39016.x

[42] D. J. Berkeland, D. A. Raymondson, and V. M. Tassin, "Tests for nonrandomness in quantum jumps," Physical Review A (Atomic, Molecular, and Optical Physics) 69, 052103 (2004). http://dx.doi.org/10.1103/PhysRevA.69.052103

[43] id Quantique, "Quantis - Quantum Random Number Generators," (2004). http://www.idquantique.com

[44] E. F. Moore, "Gedanken-Experiments on Sequential Machines," in Automata Studies, C. E. Shannon and J. McCarthy, eds. (Princeton University Press, Princeton, 1956).

[45] M. E. Gold, "Language identification in the limit," Information and Control 10, 447-474 (1967). http://dx.doi.org/10.1016/S0019-9958(67)91165-5

[46] L. Blum and M. Blum, "Toward a mathematical theory of inductive inference," Information and Control 28, 125-155 (1975).

[47] D. Angluin and C. H. Smith, "A Survey of Inductive Inference: Theory and Methods," Computing Surveys 15, 237-269 (1983).

[48] L. M. Adleman and M. Blum, "Inductive Inference and Unsolvability," The Journal of Symbolic Logic 56, 891-900 (1991). http://dx.doi.org/10.2307/2275058 
[49] M. Li and P. M. B. Vitányi, "Inductive reasoning and Kolmogorov complexity," Journal of Computer and System Science 44, 343-384 (1992). http://dx.doi.org/10.1016/0022-0000(92)90026-F

[50] A. M. Turing, "On computable numbers, with an application to the Entscheidungsproblem," Proceedings of the London Mathematical Society, Series 2 42 and 43, 230-265 and 544-546 (1936-7 and 1937), reprinted in [54].

[51] H. Rogers, Jr., Theory of Recursive Functions and Effective Computability (MacGraw-Hill, New York, 1967).

[52] P. Odifreddi, Classical Recursion Theory, Vol. 1 (North-Holland, Amsterdam, 1989).

[53] R. Landauer, "Advertisement For a Paper I Like," in On Limits, J. L. Casti and J. F. Traub, eds. (Santa Fe Institute Report 94-10-056, Santa Fe, NM, 1994), p. 39.

http://www.santafe.edu/research/publications/workingpapers/94-10-056.pdf

[54] M. Davis, The Undecidable (Raven Press, New York, 1965). 